\title{
Application of optimization to change eigenfrequency of a pinion
}

\author{
Wykorzystanie optymalizacji \\ do zmiany częstości własnych zębnika
}

JACEK STADNICKI

MICHAŁ GŁĄBEK *

DOI: https://doi.org/10.17814/mechanik.2017.7.81

\begin{abstract}
During the final stage of designing a pinion which is exploited at different rotational speeds, it is occasionally necessary to offset natural frequencies from frequencies of excitations. The way of solving this problem by means of parametric optimization of the pinion profile, assuming small changes of its shape, is discussed in the paper. The problem is solved using finite element model with regard to monolithic pinion of an aircraft gear.
\end{abstract}

KEYWORDS: mechanical engineering, modal analysis, offset of natural frequencies, parametric optimization

In high-speed flight gear, pinions are often made as monolithic, and due to the need to reduce their own weight their shafts are hollow. When designing the pinion based on the results of the verification calculations, the tooth parameters are determined and the shape and dimensions of the envelope and shaft, taking into account safety and durability criteria as well as structural considerations. Compliance with these requirements sometimes results in the design of a pinion whose natural vibration frequencies fall within the range of the excitation frequencies resulting from the rotational speed ranges occurring during the transmission operation - are in the resonant areas. This applies particularly to the gear that operates at different operating speeds, e.g. air gears.

Since the pinion meets the requirements for safety and durability and structural considerations, the question arises how to change the natural frequencies that lie in the resonant areas, or, in other words, how to offset some of the natural frequencies from the excitation frequencies. The solution to this problem inevitably leads to changes in the shape and dimensions of the toothed wheel, whose design is already at an advanced stage, such a solution is being looked for that minimally changes the design of the wheel. In practice, it points to those parts of the gear, which modification does not affect the already fulfilled the requirements of security and stability and accepted design considerations.

\footnotetext{
* Prof. dr hab. inż. Jacek Stadnicki (jstadnicki@ath.bielsko.pl), Micha Głąbek-Akademia Techniczno-Humanistyczna w Bielsku-Białej
}

\section{Modal analysis of the air gear pinion}

Pinion MES model. As a result of the modal analysis of the aerial pinion gear, we obtain a set of frequencies and forms of free (floating) vibration called the modal model. Such analysis was carried out using the finite element method in NX 9 Nastran [2]. Fig. 1 shows the pinion MES model (in view of the manufacturer's protection of the toothed wheel). Pinion modeled using eight-node, solid elements of the hexagonal type CHEXA8 (fig. 1a) and the support roller in rolling - by means of the type RBE3 nodes independent on the periphery of the opening roller in the plane of the rolling elements of the bearing and the hub dependent on the symmetry axis of the shaft (fig. 1b). At points where left and right bearings are located, pairs of coincident nodes are created that are joined by CELAS1 zero-dimensional elements of three translation strokes ( $\mathrm{X}, \mathrm{Y}$ and $\mathrm{Z}$ directions) and one rotational stiffness (around the $X$ axis). Numerical values of stiffness were assumed to be large enough $\left(1 \cdot 10^{10} \mathrm{~N} / \mathrm{mm}\right.$ and $1 \cdot 10^{10} \mathrm{~N} \cdot \mathrm{mm}$, respectively) to be considered rigid compared to the shaft. Then one of the nodes elements CELAS1 received all degrees of freedom. This way of supporting well reproduces the actual working conditions of the pinion and eliminates over-rigidness of the FEM model.

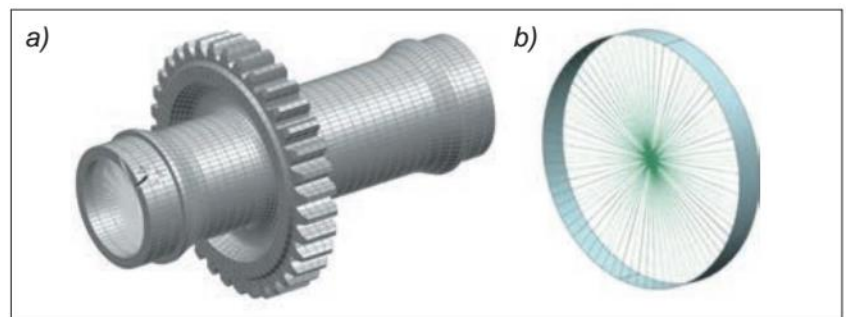

Fig. 1. MES model of the air gear pinion (a) and the bearing support model $(b)$

The developed MES pinion model has 46095 degrees of freedom. As a result of the modal analysis of the pinion MES model, we obtained a set of eigenfrequencies and eigenforms of free vibration. The values of a few of the first natural frequencies, important due to the possibility of resonance, are summarized in are shown in fig. 2. The custom frequencies are determined according to the convention $\omega_{i}^{k}$, where $i$ is the ordinal number, and $k$ is the number of nodal diameters around which the movement of rim of toothed wheel during vibration is taking place. It can be seen that the frequency pairs $\omega_{1}^{0}$ and $\omega_{2}^{0}, \omega_{4}{ }^{1}$ and $\omega_{5}{ }^{1}$ as well as 
$\omega_{7}^{2}$ and $\omega_{8}^{2}$ are the same frequencies with their corresponding same phase shifted ones. What is important for further analysis, in the pairs $\omega_{4}{ }^{1}$ and $\omega_{5}{ }^{1}$ along with $\omega_{7}^{2}$ and $\omega_{8}^{2}$, the phase shift causes rotation of the oscillation of the toothed wheel around the pinion rotation axis.

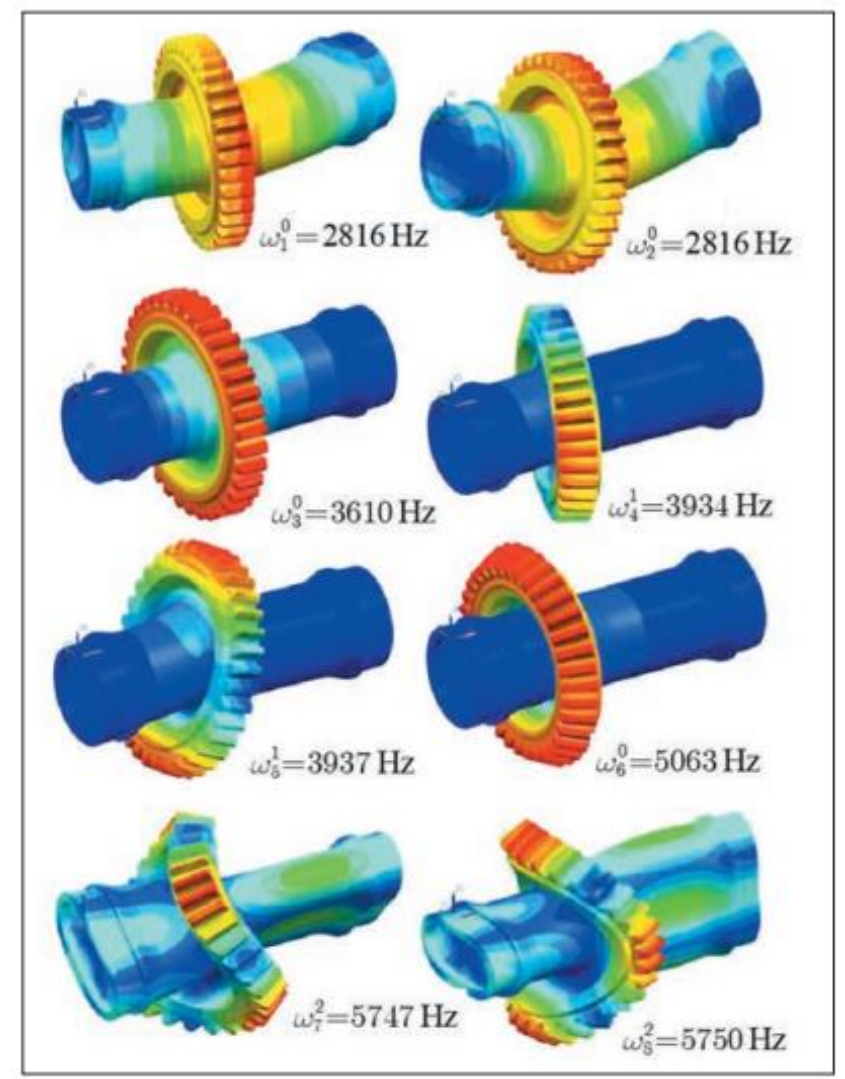

Fig. 2. Forms and frequencies of own vibrations
Campbell chart. In the engineering practice, the Campbell graph [1] is used to compare the results of the modal analysis of the rotor pinion - rotor - with its rotational velocity. Ordinate designates own frequency $\omega_{i}^{k}$ (in $\mathrm{Hz}$ ), and the abscissa - the turnover excitations $n$ (in $\mathrm{rev} / \mathrm{min}$ ). Analyzed pinion is operated at revolutions corresponding to the following operating conditions of the air gear:

- minimum revolutions: $n_{\min }=5500 \mathrm{rpm}$,

- flight: $n_{p}=8500 \mathrm{rpm}$,

- climb: $n_{w}=9500 \mathrm{rpm}$,

- maximum speed: $n_{\max }=11,000 \mathrm{rpm}$,

- speed limit $\left(105 \% n_{\max }\right): n_{g r}=11,500 \mathrm{rpm}$.

Campbell's graph is shown in fig. 3. It also shows the pinion rotation (incrementally 0 to $n_{g r}$ in $\mathrm{Hz}$ ) and the engagement line (for 35 teeth of the wheel, increasing from 0 to $35 n_{g r}$ in $\mathrm{Hz}$ ). The lines on the graph corresponding to the frequencies $\omega_{4}^{1}$ and $\omega_{7}^{2}$ do not run horizontally, but are straight pairs that are slightly inclined at a slight angle. This is caused by the rotation of the pinion around its axis. The movement of the wheel rim relative to the diameter of the junction due to vibration affects the movement of lifting - rotation of the pinion. Depending on whether these movements are consistent, whether they are opposite in phase, followed by respectively increasing or decreasing the frequency of their own. This phenomenon can be observed on the basis of the result of modal analysis. If different phaseshifted eingenforms, for which one can indicate nodal diameters, correspond to the same frequency of free vibrations of the toothed wheel (rpm), we are dealing with the change of natural frequency caused by rotations of the wheel (this effect is also called a centrifugal stiffening effect). Then in Campbell's graph, such self-frequency is represented by two straight lines from one point with the equations:

$$
\omega_{i}^{k}(n)=\omega_{i}^{k}(0) \pm k \cdot n
$$

\section{Parametric optimization of the pinion shape - resonance frequency tuning}

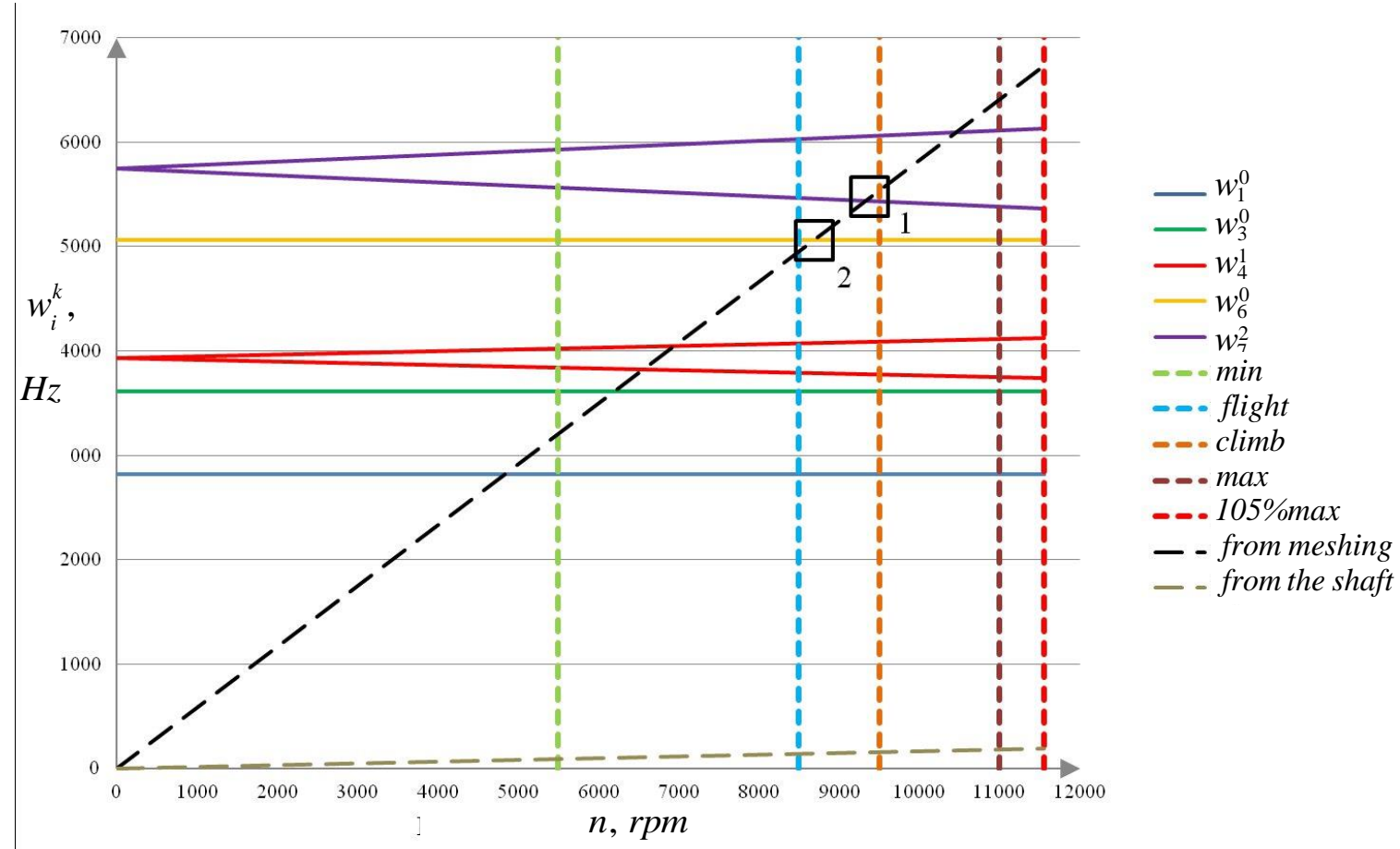

Fig. 3 Campbell Diagram for Pinion 
Diagram (fig. 3) illustrates two points (marked with squares $\square 1$ and $\square 2$ ), where there is potential risk of resonance. The lower straight resonance frequency $\omega_{7}^{2}$ crosses the straight line from the gearing at point 1 , while the straight line resonance frequency $\omega_{6}^{0}$ is at point 2 . The point 1 lies in the resonance area for the climb and point 2 for the flight. Since the resonance frequency tuning should be performed with as little interference as possible in the pinion envelope shape, the task was solved using the NX Optimizer for parametric optimization. Due to axial symmetry as parameters decision variables - values were defined defining the profile in the radial section of the pinion. Fig. 4 shows the parameters that will be changed during optimization ( $p 51$ $=x_{1}, \mathrm{p} 52=x_{2}, \mathrm{p} 53=x_{3}, \mathrm{p} 82=x_{4}, \mathrm{p} 55=x_{5}$ ), and their initial values (in $\mathrm{mm}$ ). In order to preserve the integrity of the profile, the appropriate binding is marked with the symbol O: $\mathrm{O} 1$ is a fixed point, $\mathrm{O} 2$ is the symmetry of the point along the axis $6, \mathrm{O} 3$ and $\mathrm{O} 4$ - the tangent of the curve and the symmetry of the axis 6 , and 05 is the tangent of the arc and its horizontal extension.

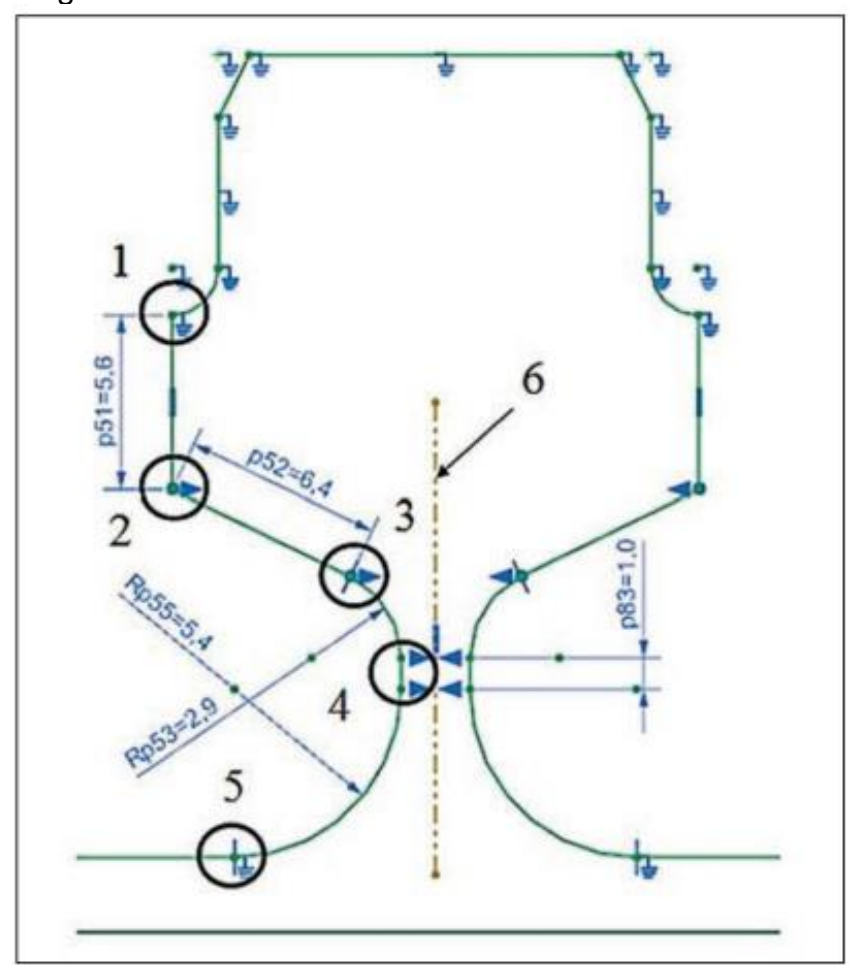

Fig. 4. Profile parameterization

Since the eigenform for eigenfrequencies $\omega_{4}{ }^{1}, \omega_{6}{ }^{0}$ and $\omega_{7}^{2}$ correspond to the deformation of the toothed wheel rim and are conjugated together, the following optimization task has been formulated:

$$
Q(\boldsymbol{x})=\omega_{4}{ }^{1}(\boldsymbol{x}) \rightarrow \text { max, gdzie } \boldsymbol{x}=\left[x_{1}, x_{2}, \ldots, x_{5}\right]^{\top}
$$

with constraints:

- $5540 \leq \omega_{7}^{2} \leq 5550 \mathrm{~Hz}$, 1 (fig. 3) halfway between the flight and climb turns,

- $\omega_{6}^{0} \leq 4700 \mathrm{~Hz}, \square 2$ (fig. 3) sufficiently offset from the rotation of the flight.

The optimal solution for the task (2) is the profile parameters (fig. 4) with the following values (the percentages of the initial values after optimization are in parentheses):

- $\mathrm{p} 51=x_{1}=5.34 \mathrm{~mm}(-4.6 \%), \mathrm{p} 52=x_{2}=5.76 \mathrm{~mm}($ $10 \%)$,

- $\mathrm{p} 53=x_{3}=2.61 \mathrm{~mm}(-10 \%), \mathrm{p} 83=x_{4}=0.98 \mathrm{~mm}(-2 \%)$, - $\mathrm{p} 55=x_{5}=5.78 \mathrm{~mm}(+7 \%)$.
Fig. 5 compares the shape of the profile before and after optimization. Changes to the profile (pinion sheath) after optimization did not violate safety criteria and also meet all design requirements.

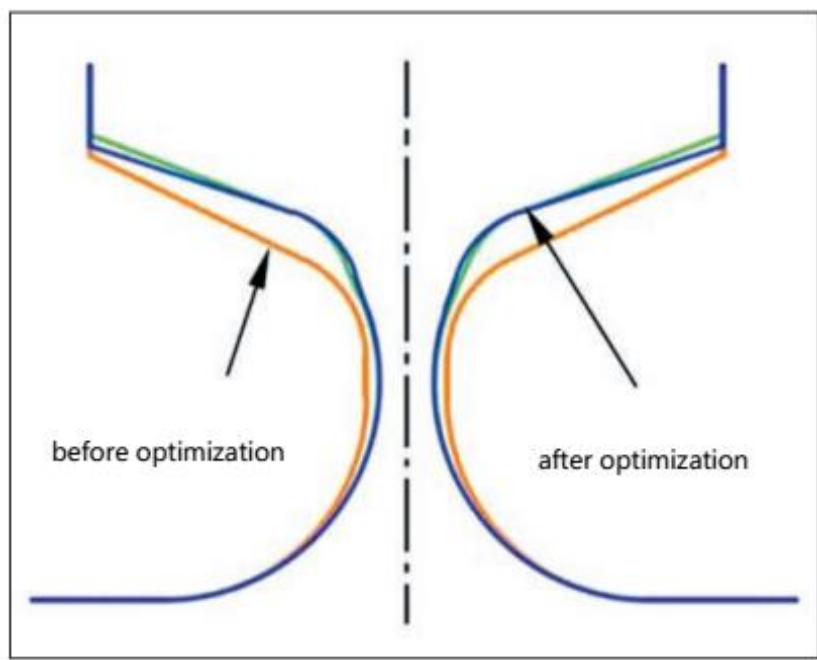

Fig. 5. Profiles before optimization and after optimization

Pinion with the optimum profile dimensions has the following own frequencies (parentheses are given in the parentheses in $\mathrm{Hz}$ relative to the value in fig. 2, which was obtained from the modal analysis of the pinion before optimization):

- $\omega_{1}^{0}=2839 \mathrm{~Hz}(+23), \omega_{3}^{0}=3613 \mathrm{~Hz}(+3)$,

- $\omega_{4}{ }^{1}=3595 \mathrm{~Hz}(-339), \omega_{6}^{0}=4688 \mathrm{~Hz}(-375)$,

- $\omega_{7}^{2}=5551 \mathrm{~Hz}(-196)$

By applying these values to the Campbell graph (fig. 3 ), it can be verified that none of the own pinion frequencies after the optimization is in the resonance area for any of the induction frequencies.

\section{Conclusions}

The problem described in the paper makes it possible to draw the following general conclusions:

- If the project is in the final stages of development and still does not meet the requirements, then you should look for parameters that change in the specified (usually small) scope will meet all requirements of the project.

- The most likely solution to the problem of a slight change in the shape of the part at the end of its design process is to provide free surfaces that are not dimensioned with fit and tolerance.

- A good method of searching for the altered shape of free surfaces in the final phase of the project is the parameterization of the profile (profile) of the part, followed by the formulation and solution of the task of optimal selection of the parameters of the contour due to the accepted criterion.

- Modern computer-aided design software provides powerful tools for parametric optimization of the MES model.

\section{REFERENCES}

1. Swanson E., Powell C.D., Weissman S. "A practical review of rotating machinery critical speeds and modes". Sound and Vibration. 39 (2005) 5: pp. 10-17.

2. http://nxtutorials.com (2016) NX9 Nastran User's Guide. 